\title{
Measurements of the Soft Gamma-ray Emission from SN2014J with Suzaku
}

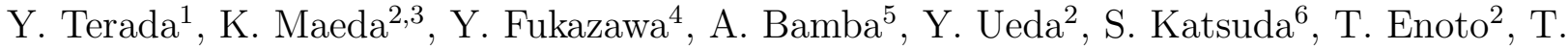

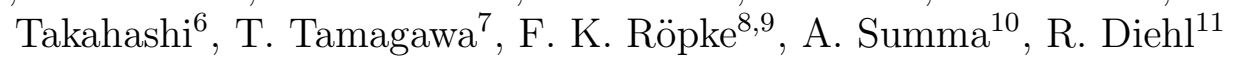

\begin{abstract}
The hard X-ray detector (HXD) onboard Suzaku measured soft $\gamma$-rays from the Type Ia supernova SN2014J at $77 \pm 2$ days after the explosion. Although the confidence level of the signal is about $90 \%$ (i.e., $2 \sigma$ ), the $3 \sigma$ upper limit has been derived at $<2.2 \times 10^{-4} \mathrm{ph} \mathrm{s}^{-1} \mathrm{~cm}^{-2}$ in the $170-250 \mathrm{keV}$ band as the first independent measurement of soft $\gamma$-rays with an instrument other than INTEGRAL. For this analysis, we have examined the reproducibility of the NXB model of HXD/GSO using blank sky data. We find that the residual count rate in the $90-500 \mathrm{keV}$ band is distributed around an average of $0.19 \%$ with a standard deviation of $0.42 \%$ relative to the NXB rate. The averaged residual signals are consistent with that expected from the cosmic X-ray background. The flux of SN2014J derived from Suzaku measurements taken in one snapshot at $t=77 \pm 2$ days after the explosion is consistent with the INTEGRAL values averaged over the period between $t=50$ and 100 days and also with explosion models of single or double degenerate scenarios. Being sensitive to the total ejecta mass surrounding the radioactive material, the ratio between continuum and line flux in the soft gamma-ray regime might distinguish different progenitor models. The Suzaku data have been examined with this relation at $t=77 \pm 2$ days, but could not distinguish models between single and double degenerate-progenitors. We disfavor explosion models with larger ${ }^{56} \mathrm{Ni}$ masses than $1 M_{\odot}$, from our $1 \sigma$ error on the $170-250 \mathrm{keV}$ X-ray flux of $(1.2 \pm 0.7) \times 10^{-4} \mathrm{ph} \mathrm{s}^{-1} \mathrm{~cm}^{-2}$.

Subject headings: stars:supernovae:general - supernovae:individual(SN2014J) - gamma rays:stars
\end{abstract}

\footnotetext{
${ }^{1}$ Graduate School of Science and Engineering, Saitama University, 255 Shimo-Ohkubo, Sakura, Saitama 3388570,Japan

${ }^{2}$ Department of Astronomy, Kyoto University, Kitashirakawa-Oiwake-cho, Sakyo-ku, Kyoto 606-8502, Japan

${ }^{3}$ Kavli Institute for the Physics and Mathematics of the Universe (WPI), University of Tokyo, 5-1-5 Kashiwanoha, Kashiwa, Chiba 277-8583, Japan

${ }^{4}$ Department of Physical Science, Hiroshima University, 1-3-1 Kagamiyama, Higashi-Hiroshima, Hiroshima 7398526, Japan

${ }^{5}$ Department of Physics and Mathematics, Aoyama Gakuin University, 5-10-1 Fuchinobe Chuo-ku, Sagamihara, Kanagawa 252-5258, Japan

${ }^{6}$ Institute of Space and Astronautical Science, Japan Aerospace eXploration Agency, 3-1-1 Yoshinodai, Sagamihara, Kanagawa 229-8510, Japan

${ }^{7}$ RIKEN, 2-1 Hirosawa, Wako, Saitama 351-0198, Japan

${ }^{8}$ Zentrum für Astronomie der Universität Heidelberg,
}

\section{Introduction}

Type Ia supernovae (SNe) are very bright stellar explosions which are detectable at optical wavelengths across cosmological distances. It is widely accepted that they originate from thermonuclear explosions of carbon-oxygen white dwarfs (WDs) in binary systems. They are among the most matured standardizable candles (Phillips et al. 1993; Riess et al. 1998; Perlmutter et al.

Institut für Theoretische Astrophysik, Philosophenweg 12, 69120 Heidelberg, Germany

${ }^{9}$ Heidelberger Institut für Theoretische Studien, Schloss-Wolfsbrunnenweg 35, 69118 Heidelberg, Germany

${ }^{10}$ Max-Planck-Institut für Astrophysik, KarlSchwarzschild-Str. 1, D-85748 Garching, Germany

${ }^{11}$ Max-Planck-Institut für extraterrestrische Physik, 85741, Garching, Germany 
1999), having a tight but phenomenologically calibrated relation between the optical peak luminosity and the decline rate of the light curve in the $B$-band.

However, the progenitors of Type Ia SNe have been poorly constrained observationally despite many on-going attempts (see e.g., Maoz, Mannucci, and Nelemos ite the strong motivation to analyze 2014, for reviews). There are several variants in terms of the ignition and propagation of the thermonuclear flame (Hillebrandt \& Niemever 2000), which can have different characteristics in (1) the evolution toward the explosion, and (2) in the mass of the exploding WD. The evolution scenarios are roughly divided into two categories referring to the nature of the progenitor systems; the single degenerate scenario (hereafter SD; Whelan \& Iben 1973; Nomoto 1982) (a C+O WD and a main-sequence/red-giant companion) or double degenerate scenario (hereafter DD; Iben \& Tutukov 1984; Webbink 1984) (a merger of two $\mathrm{C}+\mathrm{O}$ WDs). The mass of the exploding $\mathrm{WD}(\mathrm{s})$ is linked to the progenitor systems and their evolution scenario, which would affects on the cosmological usage of SN Ia as distance indicators. In the SD scenario the most popular model involves a Chandrasekhar-mass WD (e.g., Nomoto 1982). The original DD scenario is also associated with the Chandrasekhar-mass WD (e.g., Iben \& Tutukov 1984). In a recently proposed variant of the DD model, the so-called violent merger model (Pakmor et al. 2010; Röpke et al. 2012), the total mass of the ejecta (i.e., a sum of the two WDs) can exceed the Chandrasekhar-mass limit, a specific model of which is for example presented in Summa et al. (2013). Determining the ejecta mass and/or the progenitor WD is therefore of particular importance (e.g., Scalzo et al. 2014; Yamaguchi et al. 2015; Katsuda et al. 2015).

As demonstrated in the optical light curves of $\mathrm{SNe} \mathrm{Ia}$, they produce a large amount of ${ }^{56} \mathrm{Ni}$ in the explosion, on average $\sim 0.6 M_{\odot}$. Direct measurements of $\gamma$-ray emission from the decay chain, ${ }^{56} \mathrm{Ni} \rightarrow{ }^{56} \mathrm{Co} \rightarrow{ }^{56} \mathrm{Fe}$ (Arnett 1979), have been suggested to provide not only the direct evidence for the thermonuclear nature of SNe Ia Ambwani \& Sutherland 1988; Milne et al. 2004) but also various diagnostics to discriminate different models (e.g., see Maeda et al. 2012; Summa et al. 2013, for predictions based on multidimensional explosion models). Among vari- ous possibilities, it has been suggested to be a strong probe to the mass of the explosion systems (Sim \& Mazzali 2008; Summa et al. 2013), i.e., either a single Chandrasekhar-mass WD or merging two WDs for which the total mass can exceed the Chandrasekhar-mass.

the $\gamma$-ray emission from $\mathrm{SNe}$ Ia, no solid detection had been reported until 2014, including attempts for SN1991T (Lichti et al. 1994; Leising et al. 1995), SN1998bu (Georgii et al. 2002) and SN2011fe (Isern et al. 2013). The situation changed in 2014, after SN2014J was discovered on 22 January 2014 (Fossey et al. 2014) in the nearby star-burst galaxy M82 at the distance $d \sim 3.5 \mathrm{Mpc}$ (Dalcanton et al. 2009; Karachentsev \& Kashibadze 2006) and was classified as the closest Type Ia SN Avani 2014; Cao et al. 2014; Itoh et al. 2014) in the last three decades. The reconstructed date of the explosion was 14.75 January 2014 (Zheng et al. 2014). In the $\mathrm{MeV} \gamma$-ray band, the INTEGRAL satellite made possible the first detection of ${ }^{56} \mathrm{Co} \rightarrow{ }^{56} \mathrm{Fe}$ lines at 847 and $1238 \mathrm{keV}$ at $(2.34 \pm 0.74) \times 10^{-4} \mathrm{ph}$ $\mathrm{cm}^{-2} \mathrm{~s}^{-1}$ and $(2.78 \pm 0.74) \times 10^{-5} \mathrm{ph} \mathrm{cm}^{-2} \mathrm{~s}^{-1}$, respectively, in an average of the 50 to 100 days after the explosion (Churazov et al. 2014; Diehl et al. 2015). Even at earlier phases of 20 days after the explosion, the detection of ${ }^{56} \mathrm{Ni} \rightarrow{ }^{56} \mathrm{Co}$ lines at 152 and $812 \mathrm{keV}$ at $(1.10 \pm 0.42) \times 10^{-4} \mathrm{ph} \mathrm{cm}^{-2}$ $\mathrm{s}^{-1}$ and $(1.90 \pm 0.66) \times 10^{-5} \mathrm{ph} \mathrm{cm}^{-2} \mathrm{~s}^{-1}$, respectively, was reported (Diehl et al. 2014). Analysing the time evolution of ${ }^{56} \mathrm{Co}$ lines (Diehl et al. 2015; Siegert \& Diehl 2015), a ${ }^{56} \mathrm{Ni}$ mass of $0.5 M_{\odot}$ was derived. But a clear discrimination of models between SD and DD does not seem to be possible, both from limitations of the measured $\gamma$-ray intensity evolution and the theoretical prediction from different models.

These studies provided the first detection of nuclear $\gamma$-ray emission from SNe Ia, and indeed the only detection of nuclear $\gamma$-ray emission from objects beyond the local group of galaxies. This detection relies on the SPI and IBIS instruments on the same satellite INTEGRAL, and additional confirmation by a fully independent instrument is important. Moreover, while these previous reports mostly focused on the detection of the lines, a wealth of additional information is contained in the continuum emission. The $\mathrm{MeV}$ decay lines 
TABLE 1

Suzaku OBSERVATIONs TOWARDS M82

\begin{tabular}{cccccc}
\hline \hline OBSID & Target Name & $\begin{array}{c}\text { Date } \\
(\text { yyyy/mm/dd })\end{array}$ & $\begin{array}{c}\text { HXD Exposure } \\
(\mathrm{ks})\end{array}$ & $\begin{array}{c}\text { PIN Count rate } \\
\left(10^{-2} \mathrm{c} / \mathrm{s}\right)\end{array}$ & $\begin{array}{c}\text { GSO Count rate }^{\mathrm{b}} \\
\left(10^{-1} \mathrm{c} / \mathrm{s}\right)\end{array}$ \\
\hline 100033010 & M82-Wind & $2005 / 10 / 04$ & 28.6 & $1.6 \pm 0.4$ & $1.2 \pm 1.2$ \\
100033020 & M82-Wind & $2005 / 10 / 19$ & 36.1 & $2.6 \pm 0.4$ & $0.0 \pm 1.1$ \\
100033030 & M82-Wind & $2005 / 10 / 28$ & 24.0 & $3.2 \pm 0.5$ & $0.2 \pm 0.4$ \\
702026010 & M82 X-1 & $2007 / 09 / 24$ & 28.4 & $2.6 \pm 0.4$ & $0.0 \pm 0.4$ \\
$908005010^{\mathrm{c}}$ & SN 2014J & $2014 / 03 / 30$ & 193.9 & $2.6 \pm 0.1$ & $1.8 \pm 0.1$ \\
\hline
\end{tabular}

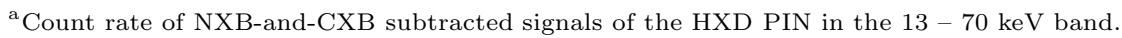

${ }^{\mathrm{b}}$ Count rate of NXB subtracted signals of the HXD GSO in the $90-500 \mathrm{keV}$ band.

' Suzaku observation in 2014 defined as 'OBS2014' in the text.

are scattered down to lower energy by Compton scattering, creating continuum emission above $\sim 100 \mathrm{keV}$ (e.g., Ambwani \& Sutherland 1988; Sim \& Mazzali 2008; Summa et al. 2013). This process is more important for more dense ejecta, unlike the line strengths which become weaker for more dense ejecta. Therefore, combining the information from the lines and the continuum, one expects to obtain additional insight into the properties of the SN ejecta that is then linked to the progenitor star. Indeed, the detection of continuum in the energy range of $200-400 \mathrm{keV}$ by INTEGRAL was reported by Churazov et al. (2014). In this paper, we report a measurement of the $\gamma$ ray continuum from SN 2014J with the Suzaku X-ray satellite (Mitsuda et al. 2007). In sections 2 and 3 and we test several explosion models to constrain the mass of ${ }^{56} \mathrm{Ni}$ and the mass of the exploding WD system in section 4 .

\section{Observation and Data Reduction}

\subsection{ToO Observation with Suzaku}

The X-ray satellite Suzaku carries two active X-ray instruments onboard (Mitsuda et al. 2007); the X-ray Imaging Spectrometer (XIS; Kovama et al. 2007) and the hard X-ray detector (HXD; Takahashi et al. 2007) to observe the $0.2-12 \mathrm{keV}$ and the $13-600 \mathrm{keV}$ bands, respectively. The HXD is a hybrid detector with PINtype Si photo-diodes for the $13-70 \mathrm{keV}$ band and phoswitch-type scintillation counters using $\mathrm{Gd}_{2} \mathrm{SiO}_{5}$ (hereafter GSO) crystals surrounded by $\mathrm{Bi}_{4} \mathrm{Ge}_{3} \mathrm{O}_{12}$ (hereafter BGO) crystals for the $60-$ $600 \mathrm{keV}$ band (Takahashi et al. 2007). It has a comparable or better sensitivity than that of $I N$ TEGRAL instruments in the $60-200 \mathrm{keV}$ band on an 'one-shot' short observation and therefore, it is suitable for our purpose to independently detect the soft $\gamma$-ray emission from SN2014J.

We triggered ToO observation of SN2014J with Suzaku from 2014 March 30 12:18 UT to 3 April $17: 23$ UT $(\mathrm{OBSID}=908005010)$, which is about $t=77 \pm 2$ days after the explosion of SN2014J, soon after the day when the sun angle allows the satellite operation. The target position was set to $(\alpha, \delta)[\mathrm{J} 2000]=\left(09^{\mathrm{h}} 55^{\mathrm{m}} 42.12^{\mathrm{s}},+69^{\circ} 40^{\prime} 26.0^{\prime \prime}\right)$ at the XIS nominal pointing position. The HXD was operated in the nominal mode; the bias voltages for one half of 64 PIN diodes were operated at 400 $\mathrm{V}$ and the other half at $500 \mathrm{~V}$, and the photomultipliers for scintillators were operated in the nominal setting of the high voltages. We also used previous observations towards the M82 region before the explosion of SN2014J for comparison in later sections. The observation in 2014J with OB$\mathrm{SID}=908005010$ (hereafter OBS2014) and previous ones are summarized in Table 1 .

\subsection{Data Reduction}

The observation data-sets were processed by the standard Suzaku pipeline version 2.8.20.35, with the calibration version (CALDBVER) of hxd20110913, xis20121106, xrt20110630, and xrs20060410 for OBS2014. In the analysis of other OBSIDs in the following Section 3, all the data are reprocessed by the ftool, 'aepipeline', with the latest CALDB files with equivalent version of OBS2014. Spectral fitting was performed with XSPEC version 12.8.1g in HEADAS 6.15.1 pack- 
TABLE 2

Systematic ERROR OF HXD-GSO NXB MODEL FOR OBS2014

\begin{tabular}{lcc}
\hline \hline ID & Energy band (keV) & Reproducibility (\%) $^{\mathrm{a}}$ \\
\hline$i$ & $86-120$ & 0.48 \\
$i i$ & $120-144$ & 0.55 \\
$i i i$ & $144-176$ & 2.37 \\
$i v$ & $176-202$ & 0.01 \\
$v$ & $202-256$ & 0.05 \\
$v i$ & $256-342$ & 0.55 \\
$v i i$ & $342-500$ & 1.80 \\
\hline
\end{tabular}

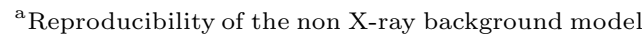
defined by the percentage between count rates of the residual and the NXB model.

age. Background was estimated from models for instrumental (i.e., 'non-X-ray') background plus cosmic diffuse X-ray background, both fitted to the SN2014J and other independent data (see below).

We did not use the XIS data, because bright X-rays from the ultra-luminous source M82 X-1 strongly contaminated the SN2014J region.

Cleaned event lists of the HXD are obtained by the standard selection criteria. The net exposure for the HXD is $193.9 \mathrm{ks}$. The non-Xray background (NXB) is estimated using the methods described in Fukazawa et al. (2009). We used the NXB events of both PIN and GSO with METHOD="LCFITDT (bgd_d)" and the version of METHODV=2.6ver1110-64. Here, if we subtract NXB events from OBS2014 data, the net count rates of PIN and GSO are $(2.6 \pm 0.1) \times 10^{-1}$ $\mathrm{c} \mathrm{s}^{-1}$ and $(1.8 \pm 0.1) \times 10^{-2} \mathrm{c} \mathrm{s}^{-1}$, respectively, in the $13-70$ or the $90-500 \mathrm{keV}$ bands, respectively. Count rates for other observations towards M82 than OBS2014 are also summarized in Table 1.

On the HXD PIN detector, the count rate of OBS2014 in Table 1 shows no significant excess over the others. According to Mivawaki et al. (2009), most of PIN signals can be considered as hard X-rays from the ULX M82 X-1, whereas $\gamma$ rays from Type Ia SNe should be weak in this energy band below $100 \mathrm{keV}$ (Maeda et al. 2012; The \& Burrows 2014). Therefore, in the following sections, we concentrate on checking the detectability of $\gamma$-rays from SN2014J with the HXD GSO in the energy band above $90 \mathrm{keV}$.

\section{Analysis and Results}

\subsection{Signal Level compared with the Sys- tematics of Non X-ray background}

The systematic error is mainly determined by the reproducibility of the NXB model, which is about $1 \%$ or less for GSO in more than $10 \mathrm{ks}$ exposure (Fukazawa et al. 2009). For OBS2014, it was confirmed with the earth occultation data during the observation, whose exposure is only $17.7 \mathrm{ks}$ with standard criteria of Cut-off-Rigidity or $21.5 \mathrm{ks}$ when we do not exclude data with bad conditions of Cut-off-Rigidity (see Table 2). We estimated a systematic uncertainties of $0.1-0.6$ $\%$ (except for one energy bins (iii) and (vii) in the table). Note that the definition of energy bins in Table 2 is determined by bins in the NXB estimation by Fukazawa et al. (2009). In order to perform a more precise check on the reproducibility of the GSO NXB models for longer exposures than $21.5 \mathrm{ks}$ during the sky observations (i.e., not earth occultation data), we estimated them with the "blank sky" observations for the HXD GSO. Among all the Suzaku observations after the launch in 2005 to 2014, we first picked up 140 observations whose exposures of the XIS exceed 120 ks, and then selected "blank sky" observations for GSO with the following criteria: (1) PIN counts in the $50-60 \mathrm{keV}$ or the $60-70 \mathrm{keV}$ bands do not exceed $3.5 \%$ of the NXB models, (2) GSO counts in the $90-500 \mathrm{keV}$ do not exceed $2.0 \%$ of the NXB models, or the number of energy bands in which GSO counts exceed $1.0 \%$ of each NXB level is less than half (i.e., 3 among 7 bands defined in Table 2), (3) the systematic errors of GSO NXB models estimated by the earth data do not exceed $2 \%$. Fi- 


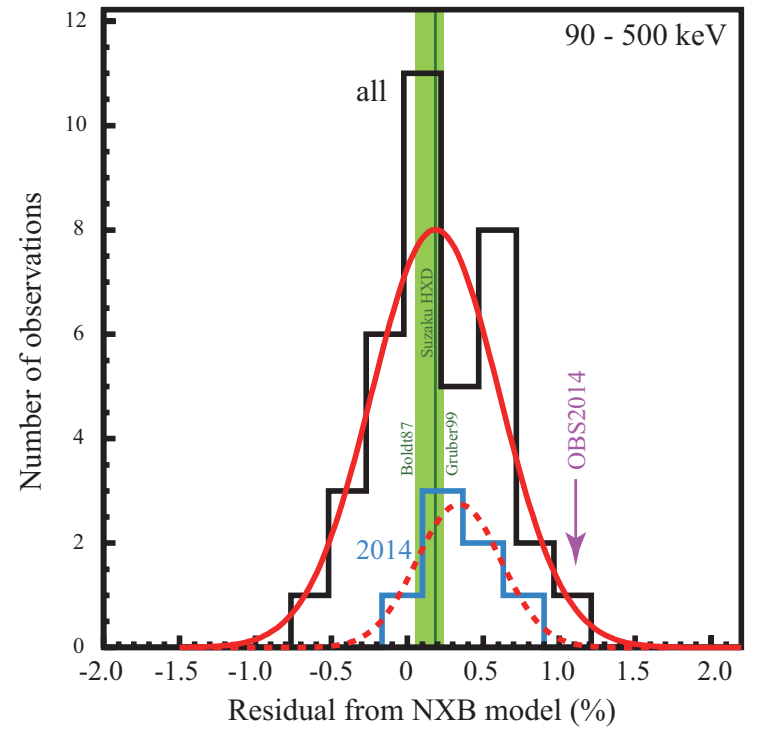

Fig. 1.- Distributions of residuals of GSO signals of blank sky observations (Table 3) from the NXB models in the $90-500 \mathrm{keV}$ band are shown in the histograms. Distribution for all the 37 observations is shown in black and that for 7 selected observations taken within 2 months before or after the SN2014J observation (i.e., OBSIDs in Table3 with note $e$ ) are in cyan. The best-fit Gaussian models for them are plotted in red. For comparison, the average value for all the blank sky observations (whose spectrum is shown in Figure 5 blue) is shown in dark green line, and the CXB levels by HEAO-1 (Boldt 1987; Gruber 1999) are shown in the green hatched box. The residual from the NXB model for OBS2014 is shown in the purple with arrow.

nally, we got 37 "blank sky" observations as listed in Table 3. The total exposure of them is 4.49 Ms. The reproducibility of the GSO NXB model for each observation is also listed in the table. As a result, the reproducibility of NXB models distributes in $0.19 \pm 0.42 \%$ for all the 37 observations or $0.34 \pm 0.28 \%$ for observations near the SN2014J date, with $1 \sigma$ errors, as demonstrated in Figure 1 . Note that this discrepancy (i.e., $0.19 \%$ offset here) between blank sky observation and NXB models comes from a contamination of CXB emission in the field of view of the HXD GSO and from the Earth's albedo emission included in NXB models; the effect is seen in green lines of Figure 1 and is numerically estimated in Section 3.2. The standard deviation $(0.42 \%)$ corresponds to $42 \%$ of the NXB-subtracted GSO signals of OBS2014 in the same energy range.

The NXB-subtracted X-ray spectra in OBS2014 are shown in Figure 22 The systematic errors of NXB models for HXD PIN and GSO are included in the plots; systematics for PIN NXB is set to be 3\% (Fukazawa et al. 2009) and those for GSO is set to values in Table 2 determined by the short earth occultation data of this observation as the worst cases. Therefore, the GSO data in OBS2014 are still significant in energy bins $(i v),(v),(v i)$ in Table 2, whereas those in previous observations towards M82 are not significant as plotted in Figure 3. In summary, we detected marginal signals from OBS2014 in the $90-500 \mathrm{keV}$ band with about $90 \%$ confidence level (i.e., about $2 \sigma$ ).

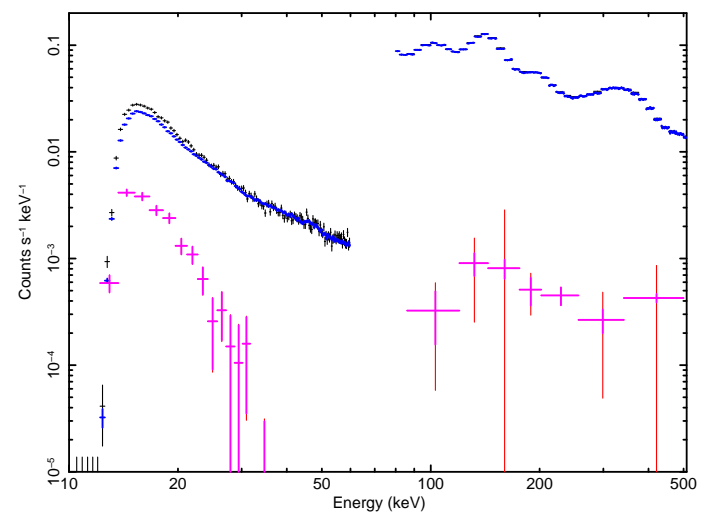

Fig. 2.- The X-ray spectra in OBS2014 with Suzaku HXD PIN (below $60 \mathrm{keV}$ ) and GSO (above $80 \mathrm{keV}$ ). Crosses in black and blue represent the raw data and the NXB models, respectively. The background subtracted spectra with statistical errors $(1 \sigma)$ are shown in magenta. Similarly, those with systematic errors of NXB models are shown in red; the systematic error for PIN is set to be $3 \%$ of non X-ray background level (Fukazawa et al. 2009) whereas those for GSO data are determined by each channel as summarized in Table 2 .

\subsection{ULX and CXB contaminations}

We now discuss in more detail the GSO signals in the three energy bins $(i v),(v),(v i)$ in Table 2. which corresponds to the $170-350 \mathrm{keV}$ band, which turn out to be most significant in Figure 2 In these GSO energy bands, any possible SN2014J 
TABLE 3

LIST OF REFERENCE OBSERVATIONS

\begin{tabular}{|c|c|c|c|c|c|}
\hline OBSID & Target Name & $\begin{array}{l}\text { Position }^{a} \\
\text { (RA,Dec) }\end{array}$ & $\begin{array}{c}\text { Obs. Date }{ }^{\mathrm{b}} \\
(\text { yyyy } / \mathrm{mm} / \mathrm{dd})\end{array}$ & $\underset{(\mathrm{ks})}{\operatorname{Exp}{ }^{\mathrm{c}}}$ & $\begin{array}{l}\text { Res. }^{\mathrm{d}} \\
(\%)\end{array}$ \\
\hline 101012010 & PERSEUS CLUSTER & $(49.9436,41.5175)$ & $2006 / 08 / 29$ & 133.2 & -0.044 \\
\hline 402015010 & LS 5039 & $(276.5633,-14.9109)$ & $2007 / 09 / 09$ & 167.7 & 0.429 \\
\hline 402033010 & SIGMA GEM & $(115.843,28.9438)$ & $2007 / 10 / 21$ & 116.2 & 0.000 \\
\hline 404001010 & AE AQUARII & $(310.0451,-0.9346)$ & $2009 / 10 / 16$ & 126.9 & -0.036 \\
\hline 408019020 & V1223 SGR & $(283.7576,-31.1629)$ & $2014 / 04 / 10^{\mathrm{e}}$ & 137.3 & 0.464 \\
\hline 408024030 & $\mathrm{~V} 2301 \mathrm{OPH}$ & $(270.1437,8.1764)$ & $2014 / 04 / 05^{\mathrm{e}}$ & 53.2 & 0.103 \\
\hline 408029010 & V1159 ORI & $(82.2495,-3.563)$ & $2014 / 03 / 16^{\mathrm{e}}$ & 177.9 & 0.157 \\
\hline 500010010 & RXJ 0852-4622 NW & $(132.2926,-45.6157)$ & $2005 / 12 / 19$ & 214.8 & -0.299 \\
\hline 502046010 & SN1006 & $(225.7268,-41.9424)$ & $2008 / 02 / 25$ & 171.4 & 0.347 \\
\hline 502048010 & 47 TUCANAE & $(6.2112,-71.9961)$ & $2007 / 06 / 10$ & 104.8 & 0.161 \\
\hline 502049010 & HESS J1702-420 & $(255.6874,-42.0709)$ & $2008 / 03 / 25$ & 131.4 & -0.076 \\
\hline 503085010 & TYCHO SNR & $(6.3139,64.1469)$ & $2008 / 08 / 04$ & 269.6 & 0.923 \\
\hline 503094010 & SNR 0049-73.6 & $(12.7817,-73.3677)$ & $2008 / 06 / 12$ & 100.7 & 0.077 \\
\hline 506052010 & G352.7-0.1 & $(261.9227,-35.1119)$ & $2012 / 03 / 02$ & 159.7 & -0.680 \\
\hline 507015030 & IC 443 & $(94.3026,22.7461)$ & $2013 / 03 / 31$ & 106.3 & 0.700 \\
\hline 508003020 & W44 SOUTH & $(284.0546,1.2208)$ & $2014 / 04 / 09^{\mathrm{e}}$ & 27.7 & -0.103 \\
\hline 508006010 & W28 SOUTH & $(270.2522,-23.558)$ & $2014 / 03 / 22^{\mathrm{e}}$ & 33.5 & 0.690 \\
\hline 508017010 & RX J1713.7-3946 NE & $(258.6449,-39.4419)$ & $2014 / 02 / 26^{\mathrm{e}}$ & 97.8 & 0.601 \\
\hline 508072010 & $0509-67.5$ & $(77.4163,-67.5163)$ & $2013 / 04 / 11$ & 154.2 & 1.006 \\
\hline 701003010 & IRAS13224-3809 & $(201.327,-38.416)$ & $2007 / 01 / 26$ & 158.5 & -0.212 \\
\hline 701031010 & MARKARIAN 335 & $(1.5539,20.2624)$ & $2006 / 06 / 21$ & 131.7 & 0.138 \\
\hline 701047010 & MRK 1 & $(19.06,33.0289)$ & $2007 / 01 / 11$ & 117.8 & 0.041 \\
\hline 701056010 & PDS 456 & $(262.0807,-14.2604)$ & $2007 / 02 / 24$ & 164.3 & -0.413 \\
\hline 702059010 & $3 \mathrm{C} 33$ & $(17.2445,13.2796)$ & $2007 / 12 / 26$ & 99.2 & 0.690 \\
\hline 703048010 & PKS $0528+134$ & $(82.7307,13.5905)$ & $2008 / 09 / 27$ & 126.4 & 0.607 \\
\hline 703049010 & $3 \mathrm{C} 279$ & $(194.0685,-5.7338)$ & $2009 / 01 / 19$ & 77.5 & 0.657 \\
\hline 704009010 & NGC 454 & $(18.511,-55.3853)$ & $2009 / 04 / 29$ & 106.0 & 0.491 \\
\hline 704062010 & NGC3516 & $(166.8656,72.6213)$ & $2009 / 10 / 28$ & 178.2 & 0.578 \\
\hline 707035020 & PDS 456 & $(262.0805,-14.2617)$ & $2013 / 03 / 03$ & 138.1 & 0.090 \\
\hline 708016010 & MKN 335 & $(1.5767,20.2085)$ & $2013 / 06 / 11$ & 116.6 & 0.140 \\
\hline 800011010 & A3376 WEST RELIC & $(90.0415,-39.9946)$ & $2005 / 11 / 07$ & 105.1 & -0.104 \\
\hline 801064010 & NGC 4472 & $(187.4441,8.005)$ & $2006 / 12 / 03$ & 96.4 & 0.253 \\
\hline 802060010 & ABELL 2029 & $(227.4644,6.0238)$ & $2008 / 01 / 08$ & 139.2 & 0.349 \\
\hline 803053010 & ABELL S753 RELIC & $(211.0241,-34.0331)$ & $2009 / 01 / 07$ & 92.3 & 0.874 \\
\hline 808043010 & FORNAX A EAST LOBE & $(51.0149,-37.2799)$ & $2013 / 08 / 02$ & 125.7 & 0.094 \\
\hline 808063010 & ESO318-021 & $(163.2697,-40.3328)$ & $2013 / 12 / 13$ & 125.2 & -0.496 \\
\hline 809119010 & ABELL2345EAST & $(321.8675,-12.1557)$ & $2014 / 04 / 30^{\mathrm{e}}$ & 83.0 & 0.161 \\
\hline
\end{tabular}

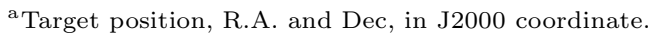

${ }^{\mathrm{b}}$ Observation start date in year/month/day.

${ }^{\mathrm{c}}$ Exposure for the HXD in ks.

${ }^{\mathrm{d}}$ Residuals of signals from NXB models in the $90-500 \mathrm{keV}$ band, shown in the percentage of the NXB.

${ }^{\mathrm{e}}$ Guest observations before or after OBS2014. 
signal could be contaminated from the ULX M82 $\mathrm{X}-1$ signal and Cosmic X-ray background (CXB) emission.
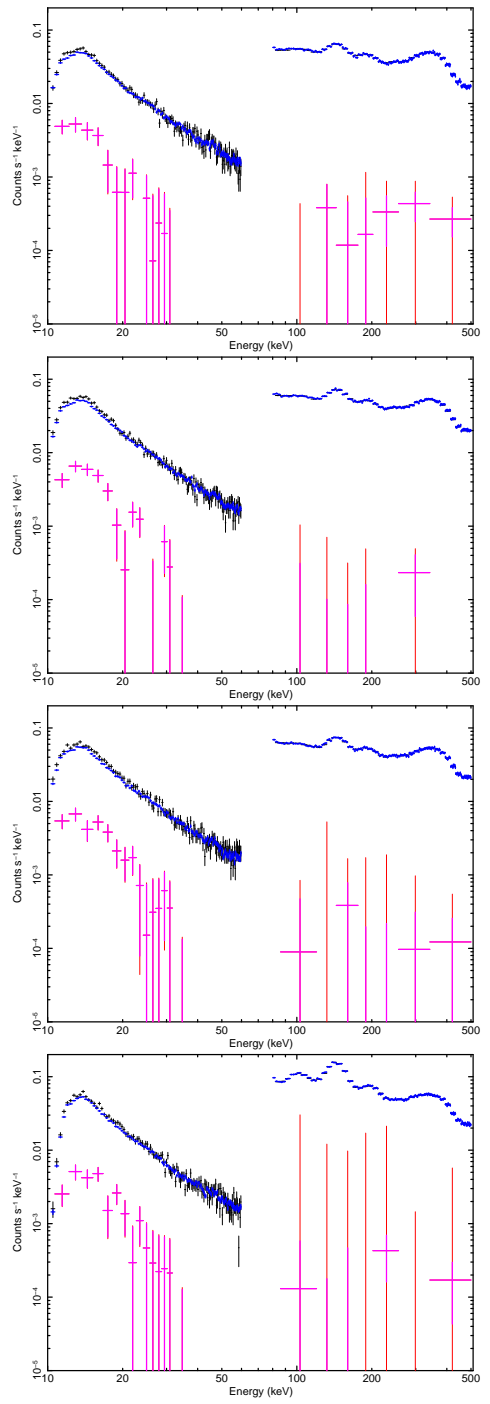

Fig. 3. - Same spectra as Figure 2, but before OBS2014 (see Table 1). Panels from the top to bottom represent the X-ray spectra in OBSID = 100033010, 100033020, 100033030, and 702026010, respectively.

The hard X-ray emission from M82 X-1 can be estimated by the direct and simultaneous measurements with HXD PIN in the $13-70 \mathrm{keV}$ band. The PIN spectrum in OBS2014 is well described by the single power law model, which is usually used for a ULX (Mivawaki et al. 2009). The best fit model has a photon index of $3.93_{-0.40}^{+0.43}$ and an X- ray flux of $1.59_{-0.08}^{+0.06} \times 10^{-11} \mathrm{erg} \mathrm{cm}^{-2} \mathrm{~s}^{-1}$ in the 13 to $70 \mathrm{keV}$ band with a reduced $\chi^{2}$ of 0.80 under 12 degrees of freedom. Instead, the multi-color disk model (Mitsuda et al. 1984) is also used to represent the ULX spectra in several phases, and is always below the power-law model in the harder Xray band. We therefore consider above power-law estimation as conservative, and the value above corresponds to the upper limit of the contribution of M82 X-1 in the GSO band by an extrapolation from the best-fit power-law model in the PIN band. In addition, the ULXs is usually variable (Mivawaki et al. 2009) as is also seen in Figure 4 . but the uncertainty on the flux from the PIN measurement here is about $5 \%$. Therefore, the contamination from ULX is about $<1.0 \pm 0.2 \%$ of the GSO signal in the $170-350 \mathrm{keV}$ band.

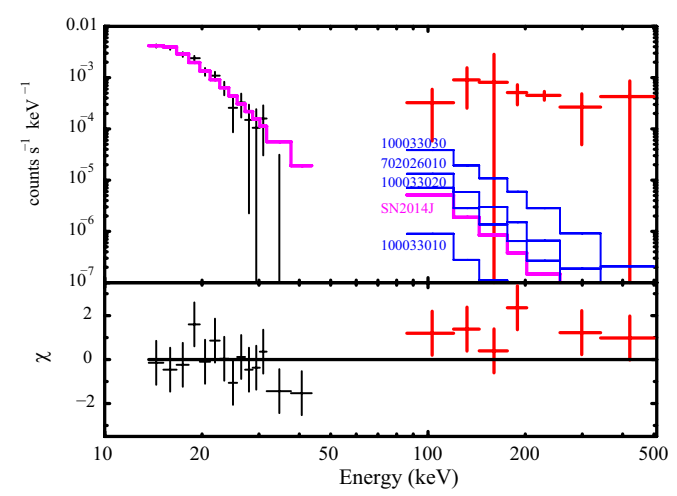

Fig. 4.- Top panel represents the same plots as red crosses of Figure 2 (i.e., PIN and GSO spectra taken in OBS2014, which are shown in black and red, respectively, in this plot), with the best-fit power-law model in magenta to reproduce the PIN data. The bottom panel shows the chi values to this model. Similarly, best-fit power-law models determined in other observations (OBSID $=100033010,100033020,100033030$, and 702026010; Mivawaki et al. 2009) are also plotted for GSO in blue.

In Figure 5, the GSO data of OBS2014 is compared with the canonical CXB model by HEAO1 (Gruber 1999), which is confirmed with the recent hard X-ray observation with Swift BAT (Ajello et al. 2008). For reference, another CXB model by Boldt (1987) is also plotted but is not valid above the $100 \mathrm{keV}$ band. The CXB models were folded into the data space using the cor- 
responding detector's angular response, which is consistent with the estimation by a full Monte Carlo simulation on Suzaku spacecraft with the Geant4 toolkit (Terada et al. 2005). The uncertainty on the angular response of GSO is checked by multiple pointing observation of Crab nebula (Kokubun et al. 2007) but is not well derived yet. Therefore, we employ two alternatives; a) pulse height spectrum estimated from CXB model by Gruber (1999) with the angular response, and b) the hard X-ray spectrum with the HXD GSO on the blank sky observations described in Section 3.1 In case $a$ ), we put $10 \%$ uncertainty on the $\mathrm{CXB}$ spectral model as described in Ajello et al. (2008). As plotted in Figure 5 the X-ray flux in $200-500$ $\mathrm{keV}$ band of these two are consistent with each other within $0.6 \sigma$ error, whereas the latter tend to have harder spectral shape (see Section 4.3 for detail). In the next section, we use both spectra for the CXB emission and then combine the two results to include systematic errors for the CXB estimation.

An additional systematic uncertainty may arise from the contribution of the Earth albedo emission in the NXB model estimated from the Earth occultation data. This is not considered in the current NXB model by Fukazawa et al. (2009). The X-ray spectrum of the Earth albedo emission can be separated from the CXB spectra by changing the coverage of the Earth within the field of view as has been done by the Swift BAT detector Ajello et al. 2008), but this method does not work for the HXD GSO in principle because of the design concept of the narrow field-of-view detector (Takahashi et al. 2007). Using the dependence of the Earth albedo level on the geomagnetic latitudes and the inclination angle $i$ of the spacecraft orbit to the Earth equator, the albedo for Suzaku at $i=31 \mathrm{deg}$ is simply interpolated between the Swift measurement (Ajello et al. 2008) at $i=20 \mathrm{deg}$ and balloon experiments at the polar and at the equator (Imhof et al. 1976). In this interpolation, we assume a systematic error of $25 \%$. Such albedo emission in the NXB model contributes to increase a signal level compared with the CXB emission, but at only about $10 \%$ of the CXB level by Gruber (1999), as plotted in Figure 5. Therefore, this causes about $1 \%$ uncertainty for the GSO signal.

In summary, we have to subtract contributions of ULX and CXB emission from the GSO signals

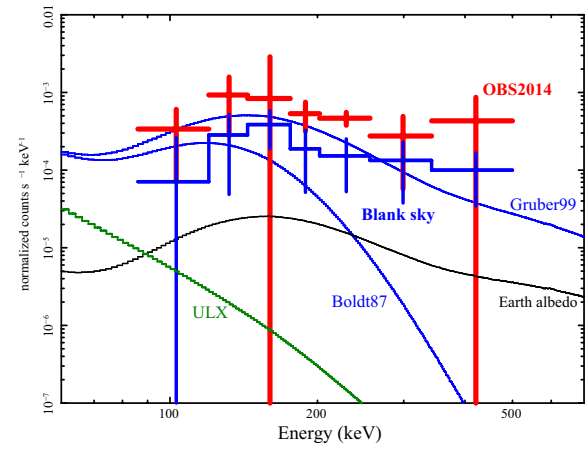

Fig. 5.- Red crosses represent the NXBsubtracted GSO spectrum for OBS2014 considering the Earth albedo emission in the NXB model (see the text). The error bar includes statistical errors and systematic errors of the NXB model and Earth albedo estimation. The Cosmic Xray backgrounds with HEAO-1 reported by Boldt (1987) and Gruber (1999) are shown in blue lines, and the Earth albedo estimated for Suzaku from the Swift and balloon experiments Ajello et al. 2008; Imhof et al. 1976) is shown in black line. X-ray spectrum of GSO taken in blank sky observations (listed in Table 3 with total exposure of $4.49 \mathrm{M} \mathrm{sec}$ ) is also plotted in blue crosses, where the error bars contain systematic errors in the NXB model and the Earth albedo. Green line represents the ULX spectrum estimated by HXD PIN (same as magenta line in Figure 44). In conversion from CXB model into the data space, we used the GSO response matrix for a flat field emission, accumulated effective areas in the Auxiliary-Response-File (ARF) database in CALDB (ae_hxd_gsoart_20051126.fits).

and add the Earth's albedo to them of OBS2014 and the blank-sky observation (not to CXB models). Numerically, the contributions of ULX, a) CXB (Gruber 1999) or b) blank sky spectrum, and the Earth albedo emission to the NXB-subtracted GSO signals (albedo emission added) are 1\%, 49\%, $39 \%$, and $3 \%$, respectively, in the $170-250 \mathrm{keV}$ band. Therefore, the GSO signal towards M82 in 2014 still remains at 4.0 or $2.5 \sigma$ significance for case $a$ ) and $b$ ), respectively, in the $170-250 \mathrm{keV}$ band i.e., energy bins $(i v)$ and $(v)$ in Table 2, even after subtraction of the ULX and CXB emissions. 


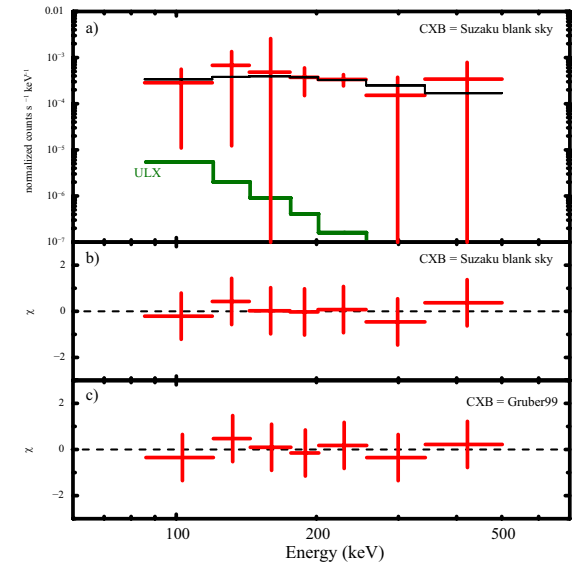

Fig. 6.- The top panel a) represents the X-ray spectrum of OBS2014 whose NXB and blank sky spectrum (blue crosses in Figure 5) are subtracted and the Earth albedo emission (black line in Figure 5D) is added. The error bars include the statistical errors and systematic errors of the NXB model and Earth albedo estimation. Contamination of ULX signals estimated by HXD PIN is shown in green (same as green line in Figure 5), which is fixed as a spectral model in the fitting. Black line shows the best-fit power-law spectrum for the GSO data, and the the chi values are plotted on the second panel b). The bottom panel c) shows the same plot as b) but the HEAO-1 result by by Gruber (1999) is used for the subtraction of Cosmic X-ray background from the GSO data in the fitting, instead of the blank sky data. In this fitting, the statistical errors and systematics of NXB model, CXB model, and the Earth albedo estimations are considered.

\subsection{Hard X-ray flux from SN2014J}

In order to derive the X-ray flux from GSO signals numerically, we performed spectral fittings with a power-law model on the GSO spectrum after the subtraction of the NXB (Section 3.1) and the CXB with consideration of the Earth albedo (Section 3.2). We tried two cases of CXB models (cases a) and b) in Section 3.2) to represent uncertainties of the CXB in the fitting. The best fit models are shown in Figure 6 and the hard Xray flux in the $170-250 \mathrm{keV}$ band is found as $\left(0.9_{-0.3}^{+0.4}\right) \times 10^{-4} \mathrm{ph} \mathrm{s}^{-1} \mathrm{~cm}^{-2}$ or $(1.6 \pm 0.4) \times 10^{-4}$ ph s${ }^{-1} \mathrm{~cm}^{-2}$ for cases $a$ ) and $b$ ) with $1 \sigma$ errors, respectively. As shown in Figure 7 the normaliza-

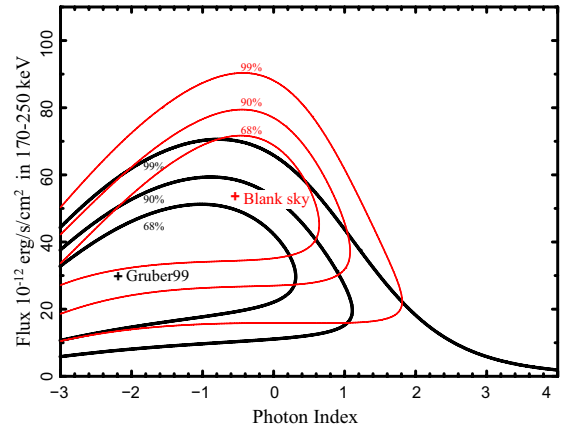

Fig. 7.- Confidence contour between the photon index and the X-ray flux in the $170-250 \mathrm{keV}$ band for the fitting of GSO data of OBS2014 in Figure 6. Red or black lines represent the contour from two fitting cases when the blank-sky data or HEAO-1 Model by Gruber (1999) are used as the Cosmic X-ray background level, respectively. The + marks show the best fit values, and the the contours indicate the $68 \%, 90 \%$, and $99 \%$ levels from the inner to the outside.

tion of the power-law model becomes zero at $99 \%$ significance level for case $a$ ) and the significance of the measured signal is about $90 \%$ confidence level (i.e., $2 \sigma$ ) in total, as already described in section 3.1. Therefore, we conclude that the Suzaku constain the X-ray flux of SN2014J to below $2.2 \times 10^{-4}$ $\mathrm{ph} \mathrm{s}^{-1} \mathrm{~cm}^{-2}$ at the $170-250 \mathrm{keV}$ band ( $3 \sigma$ limit).

\section{Discussion}

\subsection{Detection of $\gamma$-rays with Suzaku}

From the hard X-ray observation of SN2014J with Suzaku HXD at $t=77 \pm 2$ days after the explosion (Section 2), the hard X-ray flux in the $170-250 \mathrm{keV}$ band is constrained with the $99 \%$ $(3 \sigma)$ upper limit of $<2.2 \times 10^{-4} \mathrm{ph} \mathrm{s}^{-1} \mathrm{~cm}^{-2}$. This measurement complements the INTEGRAL measurements of soft X-ray band flux, at similar sensitivity obtained with a shorter exposure.

The Suzaku upper limit at $77 \pm 2$ days is consistent with those reported by INTEGRAL for the continuum emission in the $200-400 \mathrm{keV}$ band at $(2.0 \pm 0.8) \times 10^{-4} \mathrm{ph} \mathrm{s}^{-1} \mathrm{~cm}^{-2}$ at $75 \pm 25$ days 11 within errors if we correct the energy width assuming a flat spectrum as indicated by the spec-

\footnotetext{
${ }^{1}$ the value by INTEGRAL is found only in the archive (astroph/1405.3332) of Churazov et al. (2014)
} 
tral models of Maeda et al. (2012). If we take the $68 \%$ confidence levels (i.e., equivalent to the $1 \sigma$ errors) in the systematic and statistical uncertainties of the Suzaku measurement, the X-ray flux becomes $(1.2 \pm 0.7) \times 10^{-4} \mathrm{ph} \mathrm{s}^{-1} \mathrm{~cm}^{-2}$ in the same energy range. This is consistent with INTE$G R A L$ results within uncertainties. The consistency can be found in Figure 8, which shows the photon spectra estimated by the best-fit powerlaw models in cases $a$ ) and $b$ ), compared with the spectra by Churazov et al. (2015).

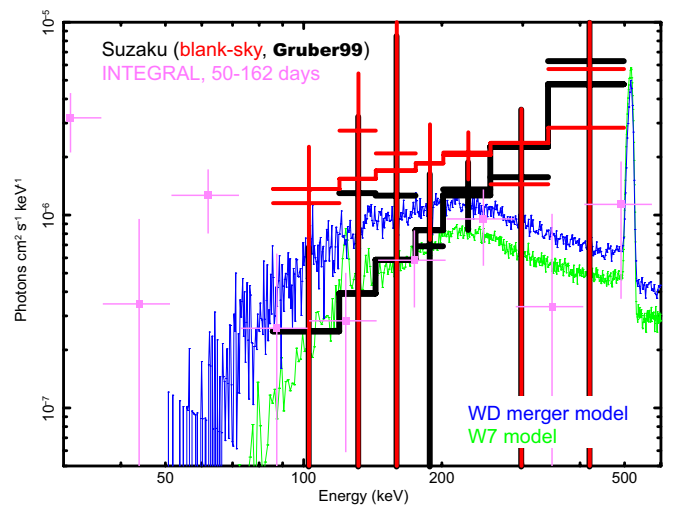

Fig. 8.- Red and black plots represent the photon spectra converted from the raw spectra in Figure 6. assuming the power-law model with the best-fit values for the data whose CXB component are set to the blank sky data (see the text) or the spectral model by Gruber (1999), respectively. Crosses and lines for them represent the data and the best-fit models, respectively. For reference, photon spectra with the INTEGRAL ISGRI at 50 - 162 days (Figure 8 blue in Churazov et al. 2015) is also plotted in magenta crosses. Green and blue lines shows the spectral models of W7 (Maeda et al. 2012) and the white dwarf merger (Summa et al. 2013), respectively, at the 75 days after the explosion with the ${ }^{56} \mathrm{Ni}$ mass of $\sim 0.6 M_{\odot}$.

\subsection{Type Ia SN models}

At $\sim 70$ days after the SN Ia explosion, decays of ${ }^{56} \mathrm{Co}$ to ${ }^{56} \mathrm{Fe}$ provide a major input into high energy radiation and thermal energy of the SN ejecta. The strongest lines are those at $847 \mathrm{keV}$ and $1238 \mathrm{keV}$. The annihilation of positrons from this $\beta+$ decay also produces either strong lines at or continuum below $511 \mathrm{keV}$. This high-energy radiation is degraded to lower energy by Compton scattering, and below $\sim 200 \mathrm{keV}$ the photons are absorbed by photoelectric absorption. These processes create characteristic continuum emission from SNe Ia in the hard X-ray and soft gamma-ray regimes.

Figure 8 shows the photon spectrum obtained by the Suzaku observation. This photon spectrum is constructed assuming a power law, and with the assumption of the best-fit power-law models either by a) the CXB model by Gruber (1999) or by $b$ ) the blank sky observations. In the same figure, the synthetic spectra of the W7 model (Maeda et al. 2012) and the violent merger model of a $0.9 M_{\odot}$ and a $1.1 M_{\odot}$ WD (Summa et al. 2013) are compared. In these models, the ${ }^{56} \mathrm{Ni}$-rich region, as well as the layers of intermediate-mass elements above the ${ }^{56} \mathrm{Ni}$-rich region, serve as the Comptonscattering layers. The W7 and delayed detonation models are (more or less) spherical, while the merger model has a large asymmetry in the distribution of the ejected material. In Figure 8, we only show the angle-averaged model spectra; the viewing angle effect is considered later. Both models have $M\left({ }^{56} \mathrm{Ni}\right) \sim 0.6 M_{\odot}$, which is consistent with what is inferred from optical properties (e.g., peak luminosity) of SN 2014J (Ashall et al. 2014).

The photon flux at $170-250 \mathrm{keV}$, taking our $2 \sigma$ signal, is indeed consistent with these models, within a systematic error related to the CXB. Above $\sim 300 \mathrm{keV}$, the nominal flux level in the Suzaku spectrum is above the level of the CXB (for both CXB models), leaving no residual SN2014J signal contribution, within uncertainties.

The most important difference in these two models is the total mass of the exploding system. The W7 model (Nomoto 1982) is a representative of an explosion of a single Chandrasekharmass WD and the expected $\gamma$-ray emission is similar to other model variants such as deflagrationdetonation models in a Chandrasekhar-mass (Maeda et al. 2012). On the other hand, in the violent merger model both of the (subChandrasekhar-mass) WDs are disrupted, leading to the super-Chandrasekhar mass for this particular model presented here (Pakmor et al. 2012; Röpke et al. 2012; Summa et al. 2013). In terms of the expected $\gamma$-ray signals, the two models are characterized by different optical depth to $\gamma$-rays through Compton scattering. The violent merger 
model has more massive ejecta and thus is more opaque (by a factor of about two), leading to a higher level of Compton continuum in the energy range of Suzaku observations. This difference is seen in Figure 8 ,

Figure 9 shows the light curves integrated in the energy range of $170-250 \mathrm{keV}$ for various models, as well as the evolution of the ratio of the same continuum flux to the $847 \mathrm{keV}$ line flux. The Suzaku upper limit and $1 \sigma$ data are also plotted as an one snapshot point at $t=77 \pm 2$ days thanks to the low-background capability of the HXD. For the ratio, we took the flux from the INTEGRAL observation at $t=75 \pm 25$ days after the explosion (Churazov et al. 2014). We adopt the energy range of $170-250 \mathrm{keV}$ since this corresponds to the marginally detected signal by Suzaku at $2 \sigma$. Shown in the figure are the W7 model, 2D delayed detonation models (Maeda et al. 2012) and the violent merger model of Pakmor et al. (2012) for which gamma-ray observables have been presented by Summa et al. (2013). The delayed detonation models were computed for different initial conditions (always with the assumption of a Chandrasekhar-mass WD), covering a wide range of $M\left({ }^{56} \mathrm{Ni}\right)$. The models with $M\left({ }^{56} \mathrm{Ni}\right)=0.47-0.72 M_{\odot}$ are indicated by yellow curves, and the $\mathrm{W} 7$ model and the violent merger model both have $\sim 0.6 M_{\odot}$ of ${ }^{56} \mathrm{Ni}$ synthesized in the explosion. These are models compatible to the optical features of SN 2014J. The emission from the violent merger model is sensitive to the viewing angle even at $\sim 60-80$ days, and thus this is shown for various viewing angles.

It is seen that for a similar amount of ${ }^{56} \mathrm{Ni}$, the violent merger model having super-Chandrasekhar mass in the total ejecta, predicts a larger flux than the models with Chandrasekhar-mass WD progenitors. This is a result of the larger optical depth as explained above. Within the observational error, all the models are consistent with the Suzaku data.

The difference between the violent merger model and the other explosion models becomes clearer in the evolution of the flux ratio. Indeed, the ratio of the continuum flux to the line flux has been suggested to be a diagnostic to distinguish the progenitor WD mass (Sim \& Mazzali 2008; Summa et al. 2013) - In case of two models producing the similar amount of ${ }^{56} \mathrm{Ni}$, a larger amount of material surrounding the radioactive isotopes (for the larger WD mass) will convert a larger fraction of the line flux to the Compton down-scattered continuum flux. Therefore the ratio of the continuum to the line flux directly mirrors the ejecta mass. This shows that it is in principle possible to constrain the total mass of the ejecta, thus the mass of the progenitor WD, through $\gamma$-ray observations. Unfortunately, the uncertainty in the Suzaku observation turned out to be too large to discriminate the models, even if we adopt the $1 \sigma$ error rather than the $3 \sigma$ upper limit. Unfortunately no constraint is obtained at a $3 \sigma$ level, but it could already start to constrain some extreme models while at a $1 \sigma$ level; the ratio predicted for some $2 \mathrm{D}$ delayed detonation models is below the Suzaku point beyond $1 \sigma$ error (i.e., the yellow lines below the data point in the lower panel of Figure 9), irrespective of the CXB model. All of these models have $M\left({ }^{56} \mathrm{Ni}\right)>1 M_{\odot}$. These models have an extended distribution in ${ }^{56} \mathrm{Ni}$, and thus have small optical depths, leading to a low ratio. We thus reject, while at a $1 \sigma$ level, the models with such a large amount of ${ }^{56} \mathrm{Ni}$ from the $\gamma$-ray signal alone fully independently of the optical emission.

\subsection{CXB measurement with the HXD}

Very few models are reported for CXB emission in an energy band above $100 \mathrm{keV}$. In Section 3.2, the hard X-ray spectrum with the HXD GSO in the $100-500 \mathrm{keV}$ band is presented in Figure 5 and compared with the canonical CXB model by Gruber (1999). In the following fittings, the Earth's albedo emission estimated in Section 3.1 is added to the NXB-subtracted spectrum of the blank sky observations. Overall uncertainties on CXB model (10\% from Ajello et al. (2008)), angular response matrix (4\% due to shade structure opaque to the sun in the X-ray mirror in Figure 11 of Terada et al. (2005)), NXB estimation (0.19\% from Section 3.1), and Earth's albedo emission (25\% from Section 3.1), are also included. If we assume that the spectral shape of CXB is given by Gruber (1999), the X-ray flux of the HXD/GSO blank-sky observation becomes $0.7 \pm 0.2$ times larger than the value of Gruber model. Numerically, it is $(2.8 \pm 0.8) \times 10^{-2} \mathrm{ph} \mathrm{s}^{-1}$ $\mathrm{cm}^{-2} \mathrm{str}^{-1}$ or $(1.3 \pm 0.3) \times 10^{-8} \mathrm{erg} \mathrm{s}^{-1} \mathrm{~cm}^{-2} \mathrm{str}^{-1}$ in the $200-500 \mathrm{keV}$ band, where the errors rep- 

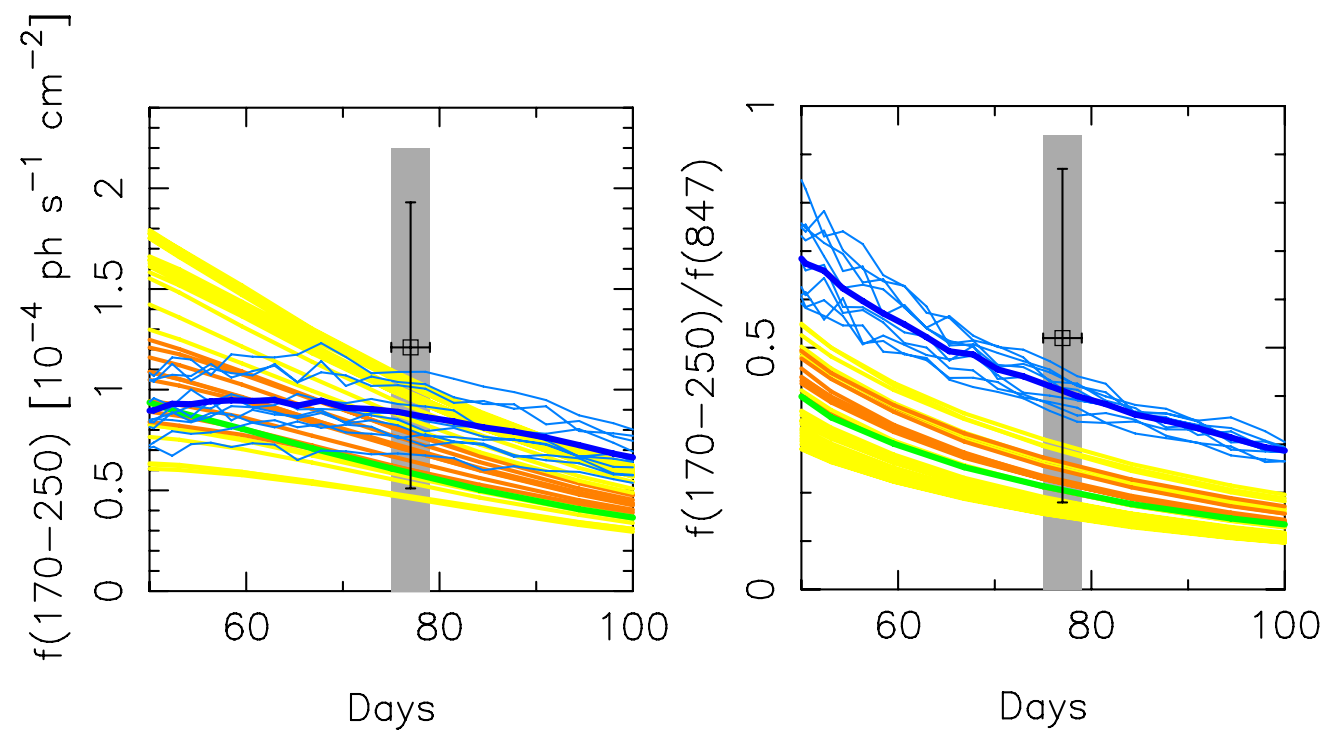

Fig. 9.- The model light curves from some SN Ia models are compared with the Suzaku observations result. The upper panel shows the flux integrated in the energy of $170-250 \mathrm{keV}$, while the lower panel shows the ratio of the $170-250 \mathrm{keV}$ flux to the $847 \mathrm{keV}$ line flux. The $3 \sigma$ upper limit is shown by a gray area, while the flux of the marginal detection is shown by an open square with an error of $1 \sigma$. The models shown here are the W7 model (green: Nomoto 1982), two-dimensional delayed-detonation models with various ignition conditions (yellow and orange: Maeda et al. 2010, 2012), and a violent merger of a $1.1 M_{\odot}$ and a $0.9 M_{\odot}$ WD (cyan and blue: Röpke et al. 2012; Summa et al. 2013). For the violent merger model, the same model viewed from different directions (cyan) are shown together with an angle-averaged emission (blue). For the delayed-detonation models, the angle-variation is not large at these epochs, and only angle-averaged behaviors are shown for 32 models covering a range of $M\left({ }^{56} \mathrm{Ni}\right)$. Models with $M\left({ }^{56} \mathrm{Ni}\right)=0.47-072 M_{\odot}$, which are compatible to observational features of SN 2014J (Ashall et al. 2014), are shown by orange curves, while the other models with larger/smaller $M\left({ }^{56} \mathrm{Ni}\right)$ are shown by yellow curves.

resent statistics only. If we reproduce the blanksky spectrum with a simple power-law model, the photon index becomes harder than that of Gruber $(1999)$ at $1.2_{-1.0}^{+1.3}$ and the X-ray flux becomes consistent with Gruber $(1999)$ at $\left(5.1_{-2.6}^{+2.5}\right) \times 10^{-2} \mathrm{ph}$ $\mathrm{s}^{-1} \mathrm{~cm}^{-2} \mathrm{str}^{-1}$ or $(2.6 \pm 1.3) \times 10^{-8} \mathrm{erg} \mathrm{s}^{-1} \mathrm{~cm}^{-2}$ $\mathrm{str}^{-1}$ in the $200-500 \mathrm{keV}$ band, whereas the Gruber model corresponds to $4.1 \times 10^{-2} \mathrm{ph} \mathrm{s}^{-1} \mathrm{~cm}^{-2}$ $\mathrm{str}^{-1}$ or $1.9 \times 10^{-8} \mathrm{erg} \mathrm{s}^{-1} \mathrm{~cm}^{-2} \mathrm{str}^{-1}$ in the same energy band. Therefore, the X-ray spectrum of the blank sky observation with GSO reproduces the CXB model by Gruber (1999) within statistical errors.

\subsection{Future Perspectives}

A next-generation X-ray satellite Hitomi (named ASTRO- $H$ before launch; Takahashi et al. 2014) has been successfully launched on 17 Feb 2016 and higher sensitivities than those of the HXD PIN/GSO or SPI/ISGRI on INTEGRAL will be achieved soon. The background level of the soft gamma-ray detector (SGD; Tajima et al. 2010; Watanabe et al. 2012; Fukazawa et al. 2014) onboard Hitomi will be reduced by one order of magnitude compared to the HXD and therefore soft $\gamma$-ray spectra from a future close-by Type-Ia $\mathrm{SNe}$ can be precisely measured as demonstrated in Maeda et al. (2012). Thus, we can distinguish the explosion models between single and double degenerate progenitors as indicated in Figure 9 In distinctions of explosion models on Figure 9 Suzaku demonstrated the importance of the snapshot measurement achieving high sensitivity in a shorter exposure ( \pm 2 days) than INTEGRAL ( \pm 25 days). In addition, we demonstrated in this paper that for future observations the refinement 
of the CXB spectral model is of critical importance.

\section{Acknowledgments}

The authors would like to thank all the members of the Suzaku team for their continuous contributions in the maintenance of onboard instruments, spacecraft operation, calibrations, software development, and user support both in Japan and the United States; especially, we would like to thank the Suzaku managers for deep understandings on the importance of this ToO observation of SN2014J with Suzaku at the late stage of mission life. The authors would like to thank H. Sano, K. Mukai, M. Sawada, T. Hayashi, T. Yuasa, H. Uchida, H. Akamatsu for giving us private datasets of Suzaku observation in the NXB and CXB studies in Sections 3.1 and 3.2. This work was supported in part by Grants-in-Aid for Scientific Research (B) from the Ministry of Education, Culture, Sports, Science and Technology (MEXT) (No. 23340055 and No. 15H00773, Y. T), a Grant-in-Aid for Young Scientists (A) from MEXT (No. 15K05107, A. B.), and a Grant-in-Aid for Young Scientists (B) from MEXT (No. 26800100, K. M.). The work by K.M. is partly supported by World Premier International Research Center Initiative (WPI Initiative), MEXT, Japan, A.S. received support from the European Research Council through grant ERC-AdG No. 341157-COCO2CASA, and FKR gratefully acknowledges the support of the Klaus Tschira Foundation.

Facilities: Suzaku, INTEGRAL, ASTRO-H, Hitomi

\section{REFERENCES}

Ajello, M., Greiner, J., Sato, G., et al. 2008, ApJ, 689, 666

Ambwani, K., ES Sutherland, P. 1988, ApJ, 325, 820

Arnett, W. D. 1979, ApJ, 230, L37

Ashall, C., Mazzali, P., Bersier, D., et al. 2014, MNRAS, 445, 4427

Ayani, K. 2014, Central Bureau Electronic Telegrams, 3792, 1

Baade, W. 1938, ApJ, 88, 285
Boldt, E., 1987, IAU Symposium, 124, 611

Cao, Y., Kasliwal, M. M., McKay, A., Bradley, A., 2014, Astronomers Telegram, 5786

Churazov, E. et al., 2014, Nature, 512406

Churazov, E., Sunyaev, R., Isern, J., et al. 2015, arXiv:1502.00255

Dalcanton, J. J., Williams, B. F., Seth, A. C., et al. 2009, ApJS, 183, 67

Diehl, R. et al., 2014, Science, 345, 1162

Diehl, R. et al., 2015, A\&3A, 574, A72

Imhof, W. L., Nakano, G. H., \& Reagan, J. B. 1976, J. Geophys. Res., 81, 2835

Itoh R., Takaki K., Ui T., Kawabata K. S., M. Y., 2014, Cent. Bur. Electron. Telegrams, 3792, 1

Karachentsev, I. D., $\&$ Kashibadze, O. G. 2006, Astrophysics, 49, 3

Kokubun, M., Makishima, K., Takahashi, T., et al. 2007, PASJ, 59, 53

Fossey, J., Cooke, B., Pollack G., et al, 2014, Central Bureau Electronic Telegrams No. 3792

Fukazawa, Y., Mizuno, T., Watanabe, S., et al. 2009, PASJ, 61, 17

Fukazawa, Y., Tajima, H., Watanabe, S., et al. 2014, Proc. SPIE, 9144, 91442C

Georgii, R., Plüschke, S., Diehl, R., et al. 2002, A\&A 394,517

Gruber, D. E. et al. 1999, ApJ, 520, 124

Hillebrandt, W., \& Niemeyer, J. C. 2000, ARA\&A, 38, 191

Iben, I., Jr., \& Tutukov, A. V. 1984, ApJS, 54, 335

Isern, J., Jean, P., Bravo, E., et al. 2013, Aछ่A, 552, $A 97$

Katsuda, S., Mori, K., Maeda, K., et al. 2015, ApJ, 808, 49

Koyama, K., et al., 2007, PASJ, 59, S23 
Leising, M. D., Johnson, W. N., Kurfess, J. D., et al. 1995, ApJ, 450, 805

Lichti, G. G., Bennett, K., den Herder, J. W., et al. 1994, A\&A, 292, 569

Lichti, G. G., Iyudin, A., Bennett, K., et al. 1996, $A \& A S, 120,353$

Maeda, K., Röpke, F.K., Fink, M., Hillebrandt, W., Travaglio, C., Thielemann, F.-K. 2010, ApJ, 712, 624

Maeda, K., Terada, Y., Kasen, D., et al. 2012, ApJ, 760,54

Maoz, D. and Mannucci, F. and Nelemans, G. 2014, ARAEA, 52, 107

Margutti, R. 2014, ApJ, 790, 52

Milne, P.A., Hungerford, A.L., Fryer, C.L., et al. 2004, ApJ, 613, 1101

Mitsuda, K., Inoue, H., Koyama, K., et al. 1984, PASJ, 36, 741

Mitsuda, K., Bautz, M., Inoue, H., et al. 2007, PASJ, 59, 1

Miyawaki, R., Makishima, K., Yamada, S., et al. 2009, PASJ, 61, 263

Nielsen, M. T. B. et al., 2014, MNRAS, 442, 3400

Nomoto, K. 1982, ApJ, 253, 798

Pakmor, R., Kromer, M., Röpke, F.K., et al. 2010, Nature, 463, 61

Pakmor, R., Kromer, M., Taubenberger, S., et al. 2012, ApJ, 747, L10

Perlmutter, S. et al., 1999, ApJ, 517, 565

Phillips, M. M. 1993, ApJ, 413, L105

Riess, A.G., Filippenko, A.V., Challis, P., et al. 1998, AJ, 116, 1009

Röpke, F.K., Kromer, M., Seitenzahl, I.R., et al. 2012, ApJ, 750, L19

Scalzo, R.A., Ruiter, A.J., Sim, S.A. 2014, MN$R A S, 445,2535$

Siegert, T., \& Diehl, R. 2015, arXiv:1501.05648
Sim, S.A., Mazzali, P.A. 2008, MNRAS,385, 1681

Sim, S. A., Fink, M., Kromer, M., et al. 2012, MNRAS, 420, 3003

Summa, A., Ulyanov, A., Kromer, M., et al. 2013, $A \& A, 554, A 67$

Tajima, H., Blandford, R., Enoto, T., et al. 2010, Proc. SPIE, 7732, 773216

Takahashi, T., et al., 2007, PASJ, 59, S35

Takahashi, T., Mitsuda, K., Kelley, R., et al. 2014, Proc. SPIE, 9144, 914425

Terada, Y., Watanabe, S., Ohno, M., et al. 2005, IEEE Transactions on Nuclear Science, 52, 902

The, L.-S., \& Burrows, A. 2014, ApJ, 786, 141

Watanabe, S., Tajima, H., Fukazawa, Y., et al. 2012, Proc. SPIE, 8443, 844326

Webbink, R. F. 1984, ApJ, 277, 355

Whelan, J., Er Iben, I., Jr. 1973, ApJ, 186, 1007

Yamaguchi, H., et al. 2015, ApJ, 801, 31

Zheng, W., Shivvers, I., Filippenko, A. V., et al. 2014, ApJ, 783, L24

This 2-column preprint was prepared with the AAS LATEX macros v5.2. 